\title{
Effects of normal or reversed proximodistal orientation of autologous nerve grafts on functional and histomorphological outcomes
}

\section{Duk Hee Lee}

Department of Emergency Medicine, Ewha Women's University Mokdong Hospital, Seoul, South Korea Jooyoung You

Department of Orthopedic Surgery, Hanyang University Guri Hospital, Guri, South Korea

Ji Won Jung

Department of Orthopedic Surgery, Hanyang University Guri Hospital, Guri, South Korea

Jung II Lee ( $D$ osjungil@gmail.com )

Department of Orthopedic Surgery, Korea University Guro Hospital, Seoul, South Korea

\section{Research Article}

Keywords: nerve, peripheral nerve, nerve repair, nerve graft, autograft

Posted Date: December 15th, 2020

DOI: https://doi.org/10.21203/rs.3.rs-125928/v1

License: (c) (i) This work is licensed under a Creative Commons Attribution 4.0 International License.

Read Full License 


\section{Abstract}

Background: Autologous nerve grafting has been considered the gold standard for the treatment of irreparable nerve gaps. However, the choice of effective proximodistal orientation of autografts (normal or reversed) is controversial. Therefore, we compared functional and histological outcomes between normal and reversed orientations of autografts.

Methods: A 10-mm section of the sciatic nerve was harvested from a donor mouse. Half the harvested nerve was grafted onto an irreparable gap in a recipient mouse using either a normal or reversed orientation. The sciatic functional index (SFI) was measured serially for up to 12 weeks postoperatively. Morphological analysis was performed using immunofluorescence staining for neurofilament (NF) and myelin protein zero (P0) in cross-sectional and whole-mount nerve preparations. Additionally, morphological analysis of the tibialis anterior muscle was performed using hematoxylin and eosin staining.

Results: The SFI recovered gradually up to 12 weeks after autografting, but there were no significant differences in the SFI between the normal and reversed orientations. The number of NF-expressing axons was significantly higher in the normal orientation than in the reversed orientation. However, there were no significant differences in the number and mean intensity of P0-expressing axons between the orientations. The cross-sectional area of myofibers was significantly larger in the normal orientation than in the reversed orientation.

Conclusions: Normally oriented autografts promote axonal regrowth and prevent neurogenic muscular atrophy compared with reverse-oriented autografts. However, despite these positive histomorphometric effects, the proximodistal orientation of the autograft does not affect functional outcomes.

\section{Introduction}

The current gold standard treatment for nerve gaps is autologous nerve grafting ${ }^{1}$. The advantages of repair using nerve autografts include provision of an immunologically inert neurosupportive scaffold and a viable source of Schwann cells to promote axonal regeneration. Recent advances have shown that several factors, including gap distance, distance from the proximal lesion to distal end target, matching the fascicle of the autograft to that of the recipient nerve, matching the diameter of autografts to that of nerve stumps, and meticulous microsurgical technique, affect axonal regeneration or functional recovery after autologous nerve grafting ${ }^{2}$. However, the choice of proximodistal orientation of the autograft (normal or reversed) is controversial.

Many surgeons believe that nerve grafting with reversed orientation has better axonal regeneration and functional recovery than normal orientation ${ }^{2}$. This belief is based on the reduced misrouting effect of regenerating axons through reversed autografts. Regenerating axons in normally oriented autografts may sprout branches in addition to the main axon of the autograft beyond the branching points, but that in reverse-oriented autografts may mitigate potential misrouting of axons. Ansselin and Davey provided the 
theoretical basis of this belief by showing that reverse-oriented autografts promoted axonal regeneration better than normally oriented autografts in a rat sciatic nerve model ${ }^{3,4}$. However, other animal studies have not shown differences between the normal or reversed orientations of autografts in functional and histological analyses ${ }^{5-10}$.

Most investigators evaluated axonal regrowth after normally or reverse-oriented autografting by counting the number of regenerating axons in the stained cross-section of the autograft. However, accurate measurement of axonal regrowth is difficult using conventional staining with thin cryostat or paraffin sections ${ }^{11}$. Therefore, some investigators used whole-mount nerve staining, which allowed that nerve or regenerating axons be visualised as a whole ${ }^{11}$.

We compared the functional and histomorphometric outcomes of normally and reverse-oriented autologous nerve grafts to investigate the effect of the proximodistal orientation of autograft on functional and neural recovery in a mouse sciatic nerve model. Histomorphometric analysis of the autograft was performed using immunofluorescence staining of the cross-sectional nerve and wholemount nerve.

\section{Results}

\section{Autograft orientation has no effect on functional recovery represented by the SFI}

All mice lost sciatic nerve function on the first postoperative day (Fig. 1). In donor mice, sciatic nerve function did not recover until 12 weeks postoperatively. In recipient mice, sciatic nerve function recovered gradually up to 12 weeks after autografting, but there was no significant difference in the SFI between normally and reverse-orientated autografts ( 12 weeks; donor, $-86.6 \pm 2.1$; normal orientation, $-59.6 \pm 4.1$; reverse orientation, $-64.5 \pm 1.9 ; P=0.29$ ). Nerve repair using autografts promoted functional recovery, but this was unaffected by the proximodistal orientation of the autograft.

\section{Cross-sectioned nerves showed that normally oriented autografts increase the total number of axons}

A cross-sectional immunofluorescence image of an uninjured nerve showed round homogenous axons enveloped with a thick round myelin sheath and a small number of DAPI-stained cells (Fig. 2A). Crosssectional immunofluorescence images of autografts showed a decreased number and size of axons, a reduced FI of myelin, and an increased number of DAPI-stained cells, which were more evident in the center than in the proximal areas of the autograft. The number of NF-expressing axons was higher in the normally oriented autograft than in the reverse-oriented autograft in the proximal ( $2776 \pm 263 \mathrm{vs.} 2115 \pm$ $121, P<0.1)$ and central areas ( $2295 \pm 85$ vs. $1585 \pm 158, P<0.05$; Fig. $2 B)$. This finding was also found for $\mathrm{P} 0$-expressing axons, but the differences were not significant (proximal, $1705 \pm 583$ vs. $1002 \pm 56, P=$ 0.35 ; central, $1356 \pm 468$ vs. $535 \pm 66, P=0.22$ ). Normally oriented autografts had a higher ratio of P0-/NF-expressing axons than reverse-oriented autografts, but this difference was not significant (proximal, $0.596 \pm 0.162$ vs. $0.474 \pm 0.003, P=0.56$; central, $0.598 \pm 0.218$ vs. $0.345 \pm 0.076, P=0.37$ ). 


\section{Whole-mount nerves showed that normally oriented autografts increase the total number of axons in the autograft and distal junction of autograft}

Although $10 \mathrm{~mm}$ of the sciatic nerve was dissected in donor mice, the empty space after nerve harvesting was filled with thin regenerating tissue. A whole-mount immunofluorescence image of this tissue showed thin fibrous tissue containing some regenerating NF-expressing axons (Fig. 3A). For all recipient mice, NFexpressing axons regrew multidirectionally to regenerate the distal side. The junctional area between the autograft and the distal stump of the recipient nerve in mice treated with normally oriented autograft showed that numerous NF-expressing axons regrew crossing the junctional area to the distal stump with circumferential regrowth from the peripheral surface of the distal stump. There were numerous bluecolored DAPI-positive cells in the distal stump (Fig. 3B). The junctional area in mice treated with reverseoriented autograft showed that most NF-expressing axons regrew longitudinally, crossing the junction toward the distal stump. There was minimal circumferential regrowth from the peripheral area of the distal stump (Fig. 3C).

A total of five images per nerve (two proximal, one central, and two distal areas of the autograft) were analyzed to quantify the number of NF-expressing axons and the mean FI of P0-expressing axons. The number of NF-expressing axons in the proximal area of the autograft was not different between normally and reverse-oriented autografts, but it was higher in the central or distal area of the normally oriented autograft (central, $1247 \pm 118$ vs. $808 \pm 87$; Distal- $1,1216 \pm 107$ vs. $839 \pm 62$; Distal-2, $1214 \pm 85$ vs. $888 \pm$ 49; $P<0.05$; Fig. 4). The mean Fl of P0 expression in each of the five areas of the nerve was not different between either orientation and it was higher in the distal area of the normally oriented autograft than in the reversed- oriented autograft, but the difference was not significant.

\section{CSA of TA myofibers after normally oriented autografting is larger than that after reverse autografting}

Denervation-induced muscle atrophy was observed in donor mice, but autografting alleviated muscle atrophy in recipient mice (Fig. 5). The wet weight ratio of the TA muscle and quantitative analysis of TA myofibers in donor mice were significantly less than those in recipient mice $(P<0.01)$. The CSA of myofibers was larger in normally oriented autografts than in reverse-oriented autografts $(P<0.05)$, which indicated that the protective effect from denervation-induced muscle atrophy was better after normally oriented autografting than that after reverse-oriented autografting. However, there were no significant differences in the wet weight ratio and MFD of myofibers between either orientations.

\section{Discussion}

In total, eight animal studies using rat or rabbit models have been performed to investigate the effect of autograft orientation on neural and functional regeneration during interpositional nerve grafting, with most conducted $>2-3$ decades ago ${ }^{3-8}$. Recent studies have used advanced tools to assess outcomes after autografting, including immunohistochemical staining of cross-sectioned nerves using antibodies against choline acetyltransferase or $\mathrm{NF}$, and diffusion tensor imaging of the nerve $\mathrm{e}^{9,10}$. In our study, we 
used behavioral evaluation of sciatic nerve function, morphometric analysis using immunohistochemicalstained cross-sectioned and whole-mount nerves, and morphometric analysis using H\&E-stained crosssectioned muscles to investigate the effect of autograft orientation on nerve regrowth. Immunohistochemical staining with whole-mount nerves is useful for investigating axon path finding after autogenous nerve grafting, unlike staining cross-sectioned nerves ${ }^{11}$. Because prevention of denervation-induced muscle atrophy could potentially improve functional outcomes after nerve grafting, assessment of the degree of muscle atrophy is also useful to evaluate the effect of autograft orientation ${ }^{12}$.

Most studies did not find significant differences between normally or reverse-oriented autografts f-10 $^{5}$. However, Ansselin and Davey suggested that reverse-oriented autografts may facilitate axonal regeneration better than normally oriented autografts ${ }^{3,4}$. Their studies laid the grounds for proponents of using reverse autografts with branches. They presented the axon counts in distal autograft as two values; absolute number and percentage values compared to proximal autografts. The former values were similar between normally or reverse-oriented autografts, but the latter values in reverse-oriented autografts was higher than in normally oriented autografts. They supposed that termination of collateral sprouts of normally oriented autograft consequently resulted in a lager reduction in axon counts and overall regeneration. However, our histomorphological analysis revealed that the number of NF or POexpressing axons and ratio of P0-/NF-expressing axons were higher in normally oriented autografts than in reverse-oriented autografts. Our results suggest that interpositional nerve grafts using normally oriented autografts promote better axonal regrowth than those using reverse-oriented autografts. Maintaining normal physiological orientation may provide better chances of axonal regeneration to maintain physiologic axoplasmic flow ${ }^{13}$.

Our confocal microscopic findings using whole-mount immunofluorescence staining demonstrated that numerous axons sprouted though autograft into the distal stump, which was more obvious in normally oriented autografts than in reverse-oriented autografts. Moreover, in the junctional area between these autografts and the distal stump, the regenerating axons regrew not only longitudinally but also circumferentially. The regenerating axons in reverse-oriented autografts sprouted only longitudinally without circumferential regrowth. These are unique findings because no studies have attempted immunofluorescence staining of whole-mount nerves. We hypothesized that axons regrow through the normal orientation of the sciatic nerve, which has three divisions including the tibial, peroneal and sural nerve, and regenerating axons through the other two divisions, excluding the main division, try to connect to each other. Thus, these connecting axons look like circumferential regrowth of axons. However, in reverse-oriented autografts, axons regrow and follow only the main division; thus, there is no circumferential regrowth.

However, improvement in quantitative axonal counts and qualitative histological morphology are not synonymous with functional improvement. Our functional results, represented by the SFI, revealed no significant differences between normal and reversed orientations, consistent with results of previous 
studies evaluating the $\mathrm{SFI}^{4,7,9}$. Although our histomorphometric analysis of the TA muscle showed that normally oriented autografts had a higher CSA than the reverse-oriented autograft, indicating that normal orientation better prevented neurogenic atrophy; however, this positive effect could not be connected with functional improvement. When all studies were combined, there was no difference in functional recovery between normally and reverse-oriented autografts, regardless of which orientation had more axonal counts in the grafts.

Our study has some limitations. First, we did not evaluate the ultrastructural features of the nerve, such as myelin thickness and diameter of the myelinated axon, which would have been helpful to assess neural regeneration according to nerve orientation. Second, although walking track analysis to calculate the SFI has been considered the gold standard to evaluate function after sciatic nerve injury ${ }^{14,15}$, it may be not sensitive enough to detect subtle changes in functional recovery between normally and reverseoriented autograft groups ${ }^{4}$. In our study, the SFI differences between groups were very small to show statistical significance. Nonetheless, the evaluation of functional status cannot be understated because the primary treatment goal is not histological improvement, but functional improvement.

Overall, the results suggest that interpositional nerve grafting using normally oriented autografts better promotes axonal regrowth and prevents neurogenic muscular atrophy than reverse-oriented autografts. Our confocal microscopic findings demonstrated that axonal regrowth patterns are different according to nerve orientation. Despite positive histomorphometric effects of normal nerve orientation, the autograft orientation does not affect functional outcomes.

\section{Methods}

\section{Experimental design}

The experimental design and surgical protocols were approved by the Institutional Animal Care and Use Committee (IACUC) at the Hanyang University College of Medicine (HY-IACUC-20-0031). The mice were housed at the animal facility and the experimental animals were handled according to the IACUC guidelines for the care and use of laboratory animals. This study was carried out in compliance with the ARRIVE guidelines.

Ten-week-old female C57BL/6J mice weighing 20-25 g were used. Mice were assigned to the donor, normally oriented autograft, and reverse-oriented autograft groups ( $n=10$ per group; Fig. 6). In the donor group, $10 \mathrm{~mm}$ of the sciatic nerve from the right hind limb was harvested as an autograft in recipient mice. This group also served as a negative control. In the recipient mice groups, a nerve gap was created by dissecting $3 \mathrm{~mm}$ of the sciatic nerve, which was then repaired using half ( $5 \mathrm{~mm}$ ) of the dissected donor nerve section in either the normal or reversed proximodistal orientation. When the graft was placed and repaired in the original orientation, proximal to distal, the orientation was called normal, whereas grafting in the distal to proximal orientation was called the reversed orientation. 
After surgery, motor functional recovery was monitored by walking track analysis at serial time points up to 12 weeks after injury. After 12 weeks, the sciatic nerve was harvested, and morphological analysis of the nerve was performed using immunofluorescence staining for neurofilament (NF) heavy chain and myelin protein zero (P0) in cross-sectional and whole-mount nerve preparations. Additionally, bilateral tibialis anterior (TA) muscle was harvested, weighed in grams, and morphological analysis of the TA muscle fibers was performed using hematoxylin and eosin (H\&E) staining.

\section{Animal model}

The right sciatic nerve was exposed through the transgluteal approach with sterile manner. In the donor mice, $10 \mathrm{~mm}$ of the sciatic nerve from the right hind limb was harvested using a 10-mm jig and microscissors and kept in cold phosphate-buffered saline (PBS). For recipient mice, a nerve gap was created by dissecting $3 \mathrm{~mm}$ of the sciatic nerve using a 3-mm jig and microscissors. As both resected ends retracted owing to the elastic recoil of the perineural tissue and the harvested nerve inevitably shrunk in length, the autograft harvested should be $₫ 25 \%$ longer than the recipient nerve gap to compensate for these changes ${ }^{1,13}$ Half $(5 \mathrm{~mm})$ the dissected nerve section from donor mice was placed in the nerve gap of recipient mice, either in the normal or reversed proximodistal orientation. The autograft was kept well aligned and coapted with fibrin sealant (GC Pharma, Seoul, South Korea) at both resected ends under an operating microscope. The skin incision was closed using 4 or 5 interrupted 4-0 Prolene sutures (Ethicon, Somerville, NJ). Mice were then returned to their cages, allowed free activity, and were followed up under supervision of the attending veterinarian.

\section{Walking track analysis}

Walking track analysis was performed according to a previously described method ${ }^{14,15}$ to quantify the sciatic functional index (SFI) noninvasively to evaluate motor functional recovery after sciatic nerve injury. Two independent interpreters blinded to study protocols selected three footprints per limb, which were measured using Vernier calipers.

\section{Immunofluorescence staining of cross-sectioned nerves}

Twelve weeks postoperatively, mice were euthanized by $\mathrm{CO} 2$ asphyxiation with cervical dislocation. The full length of the sciatic nerve containing the autograft was harvested and fixed in $4 \%$ paraformaldehyde (PFA) at $4^{\circ} \mathrm{C}$ overnight. The nerves were embedded in paraffin, and cross-sections were taken from the proximal and central portions of the autograft. Cross-sections $(5 \mu \mathrm{m})$ were taken from the embedded blocks and mounted on slides. The sections were deparaffinized and serially rehydrated using xylene and ethanol, respectively. Antigen retrieval, permeabilization and blocking of nonspecific binding was performed. Primary antibody staining was performed with anti-NF heavy chain antibody (1:500, ab4680; Abcam) and anti-P0 antibody (1:200, ab31851; Abcam) in 5\% BSA. Incubation with 4,6-diamidino-2phenylindole (DAPI, 1:1000, ab228549; Abcam) and fluorescent secondary antibodies (Alexa Fluor 488 and 594-conjugated antibodies [1:500, ab197485 and ab150088, Abcam]) was performed after washing the sections in PBS to remove the primary antibodies. The nerve sections were examined using a Leica 
DMI4000B fluorescence microscope (Leica Microsystems, Wetzlar, Germany). Four stained nerve sections per group were analyzed in a semiautomatic fashion using ImageJ (National Institutes of Health, Bethesda, MD) to determine the number of NF- or P0-expressing axons.

\section{Immunofluorescence staining of whole-mount nerves}

Whole-mount immunofluorescence staining of the nerve was performed according to our previous method and that reported in another study ${ }^{11,16}$. After euthanasia, the full length of the sciatic nerve was harvested and fixed overnight in $4 \%$ PFA at $4^{\circ} \mathrm{C}$. The nerves were then washed three times for 10 min each with PTX (PBS with $1 \%$ Triton X-100 [Sigma X100]) and incubated in a blocking solution ( $10 \%$ normal goat serum in $5 \%$ BSA PTX) overnight at $4^{\circ} \mathrm{C}$. The following day, the nerves were transferred into two primary antibodies (NF, 1:1000; P0, 1:500) in 5\% BSA PTX and incubated for $72 \mathrm{~h}$ at $4^{\circ} \mathrm{C}$ with gentle rocking. After incubation, the nerves were washed with PTX for $4 \mathrm{~h}$ at $4^{\circ} \mathrm{C}$, with hourly PTX change. Fluorescent secondary antibodies (Alexa Fluor 488 and 594-conjugated antibodies [1:500]) and DAPI (1:1000) were incubated with the nerves for $48 \mathrm{~h}$ at $4^{\circ} \mathrm{C}$ with gentle rocking. Subsequently, the nerves were washed with PTX three times for 15 min each, followed by a 4-h wash with PTX, with hourly PTX change. The nerves were then washed overnight without changing PTX at $4^{\circ} \mathrm{C}$. The next day, nerves were washed with PBS three times for 10 min each to remove triton and cleared sequentially in $25 \%$ and $50 \%$ glycerol (Sigma G6279) in PBS for 6 and 12 h, respectively, for each glycerol concentration. After clearance, the nerves were mounted in anti-fade fluorescence mounting medium (ab104135, Abcam). Stained whole nerves were examined, and the proximal, central, and distal portions of the autograft were imaged using a Leica TCS SP5 confocal microscope (Leica Microsystems). Five stained whole-mount nerves per group were analyzed automatically using Leica Application Suite X (LAS X) (Leica Microsystems) to determine the number of NF-expressing axons and the fluorescence intensity (FI) of POexpressing myelin. Perpendicular lines of nerve fibers were drawn on the nerve. The plot and mean values of $\mathrm{FI}$ were generated automatically according to the $\mathrm{FI}$ on the perpendicular lines using LAS X. We used the number of peaks and mean values of FI for plotting the NF- and PO-stained images, respectively.

\section{H\&E staining of muscles}

After euthanasia, the TA muscles of the bilateral hind limbs were harvested. The wet muscle weights were obtained and presented as wet weight percentage of the uninjured side. The harvested muscles were immersed in 4\% PFA, embedded in paraffin, cross-sectioned longitudinally at $5 \mu \mathrm{m}$, stained with $\mathrm{H} \& \mathrm{E}$, and imaged using a BX53 microscope with DP72 camera (Olympus, Center Valley, PA). Four random microscopic fields were chosen from each muscle and four animals were analyzed per group. Quantitative analysis for the cross-sectional area (CSA) and minimum Feret's diameter (MFD) of muscle fibers was performed using ImageJ.

\section{Statistical analysis}

All results are expressed as mean \pm standard error of mean. Data were analyzed by two-tailed Student's ttest and plotted using GraphPad PRISM 8 (GraphPad Software, San Diego, CA), with $P<0.05$ considered 
significant.

\section{Declarations}

Acknowledgments: The authors would like to thank Kyung Jun Lee and Se Mi Park from Korea University Guro Hospital, Seoul, South Korea for the excellent technical assistance. This work was supported by the National Research Foundation of Korea (NRF) grant funded by the Korea government (MSIT) (NRF2020R1C1C1004851).

\section{Author contributions}

Design of the study, data acquisition and interpretation, and writing initial draft: L.D.H; Data analysis: Y.J.; Data analysis: J.J.W; Concept and design of the study, funding acquisition, data organization and interpretation, revising and editing the draft with intellectual content: L.J.I. All authors approved the final manuscript.

\section{Competing interests}

The authors declare no competing interests.

\section{References}

1. Griffin, J. W., Hogan, M. V., Chhabra, A. B. \& Deal, D. N. Peripheral nerve repair and reconstruction. J Bone Joint Surg Am 95, 2144-2151, doi:10.2106/JBJS.L.00704 (2013).

2. Roberts, S. E. et al. To reverse or not to reverse? A systematic review of autograft polarity on functional outcomes following peripheral nerve repair surgery. Microsurgery 37, 169-174, doi:10.1002/micr.30133 (2017).

3. Ansselin, A. D. \& Davey, D. F. Axonal regeneration through peripheral nerve grafts: the effect of proximo-distal orientation. Microsurgery 9, 103-113, doi:10.1002/micr.1920090210 (1988).

4. Ansselin, A. D. \& Davey, D. F. The regeneration of axons through normal and reversed peripheral nerve grafts. Restor Neurol Neurosci 5, 225-240, doi:10.3233/RNN-1993-5307 (1993).

5. Sanders, F. K. \& Young, J. Z. The degeneration and re-innervation of grafted nerves. $J$ Anat 76, 143166147 (1942).

6. Stromberg, B. V., Vlastou, C. \& Earle, A. S. Effect of nerve graft polarity on nerve regeneration and function. J Hand Surg Am 4, 444-445, doi:10.1016/s0363-5023(79)80039-8 (1979).

7. Sotereanos, D. G. et al. Reversing nerve-graft polarity in a rat model: the effect on function. $J$ Reconstr Microsurg 8, 303-307, doi:10.1055/s-2007-1006712 (1992).

8. Nakatsuka, H. et al. Experimental study of polarity in reversing cable nerve grafts. J Reconstr Microsurg 18, 509-515, doi:10.1055/s-2002-33323 (2002). 
9. Afshari, A. et al. Assessment of the Effect of Autograft Orientation on Peripheral Nerve Regeneration Using Diffusion Tensor Imaging. Ann Plast Surg 80, 384-390, doi:10.1097/SAP.0000000000001357 (2018).

10. Kim, J. et al. Nerve Repair and Orthodromic and Antidromic Nerve Grafts: An Experimental Comparative Study in Rabbit. Biomed Res Int 2020, 5046832, doi:10.1155/2020/5046832 (2020).

11. Dun, X. P. \& Parkinson, D. B. Visualizing peripheral nerve regeneration by whole mount staining. PLoS One 10, e0119168, doi:10.1371/journal.pone.0119168 (2015).

12. Yue, L. et al. 4-Aminopyridine attenuates muscle atrophy after sciatic nerve crush injury in mice. Muscle Nerve 60, 192-201, doi:10.1002/mus.26516 (2019).

13. Wolford, L. M. \& Stevao, E. L. Considerations in nerve repair. Proc (Bayl Univ Med Cent) 16, 152-156, doi:10.1080/08998280.2003.11927897 (2003).

14. Bain, J. R., Mackinnon, S. E. \& Hunter, D. A. Functional evaluation of complete sciatic, peroneal, and posterior tibial nerve lesions in the rat. Plast Reconstr Surg 83, 129-138, doi:10.1097/00006534198901000-00024 (1989).

15. Varejao, A. S., Meek, M. F., Ferreira, A. J., Patricio, J. A. \& Cabrita, A. M. Functional evaluation of peripheral nerve regeneration in the rat: walking track analysis. J Neurosci Methods 108, 1-9, doi:10.1016/s0165-0270(01)00378-8 (2001).

16. Lee, J. I., Hur, J. M., You, J. \& Lee, D. H. Functional recovery with histomorphometric analysis of nerves and muscles after combination treatment with erythropoietin and dexamethasone in acute peripheral nerve injury. PLoS One 15, e0238208, doi:10.1371/journal.pone.0238208 (2020).

\section{Figures}




\section{Sciatic Functional Index}

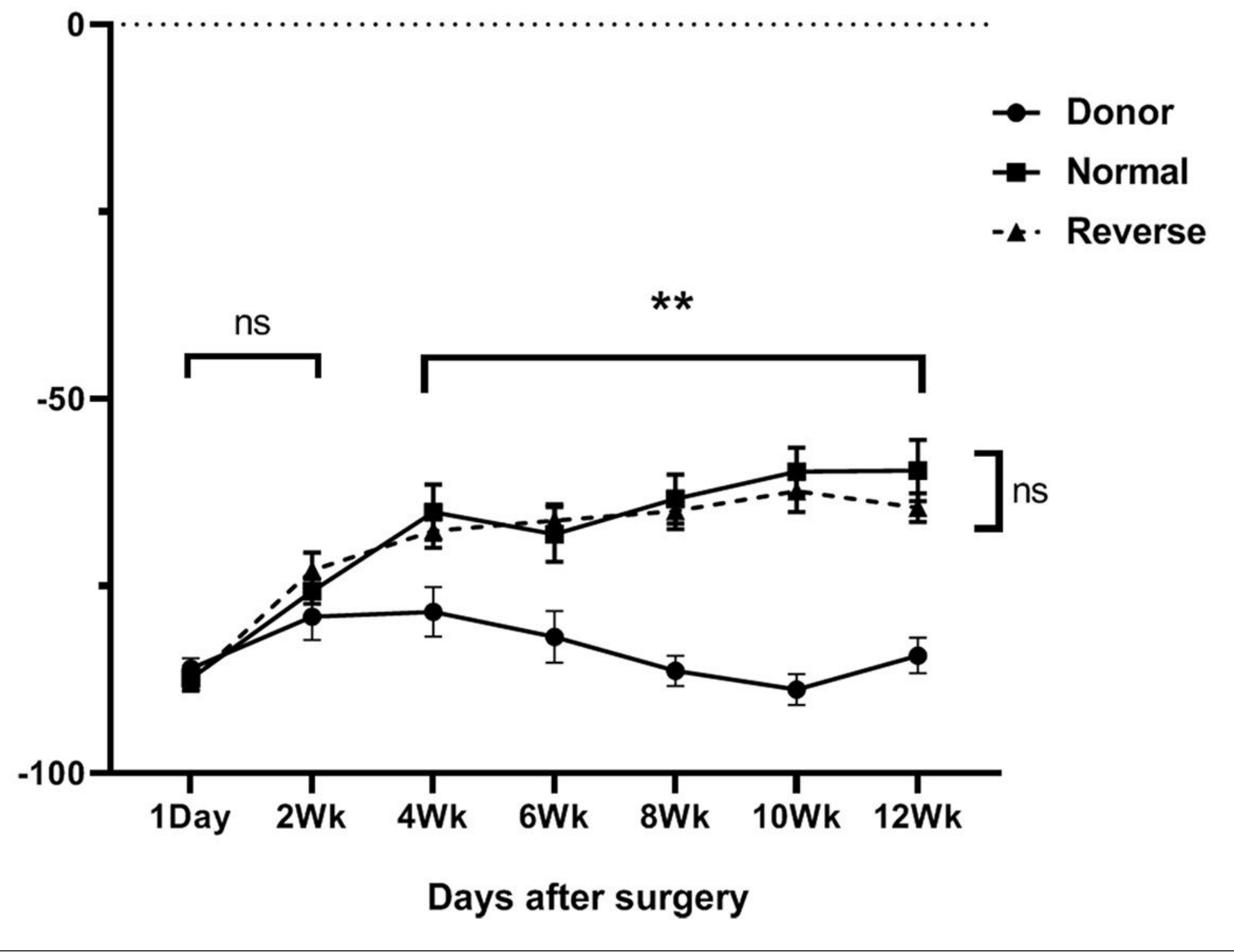

Figure 1

Functional improvement after interpositional autogenous nerve grafting for irreparable nerve defects in mice. All mice including donor and recipient mice lost sciatic nerve function completely on the first day after surgery, and sciatic nerve function recovered gradually up to 12 weeks after interpositional autogenous nerve grafting in recipient mice. Mice treated with normally oriented autografts had a higher sciatic functional index than those treated with reverse-oriented autografts at 12 weeks postoperatively, but this difference was not statistically significant ( $n=10$ /group; $n s$, no significance; ${ }^{\star \star} P<0.05$; Donor, donor mice; Normal, normally oriented autograft; Reverse, reverse-oriented autograft). 
Normal Orientation

Uninjured

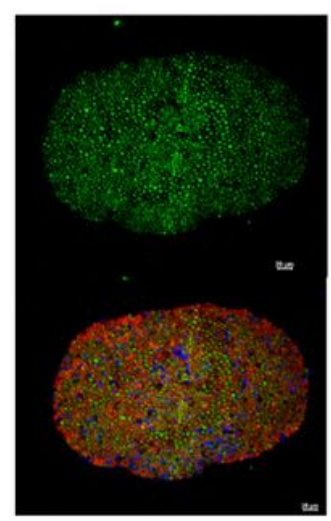

Proximal

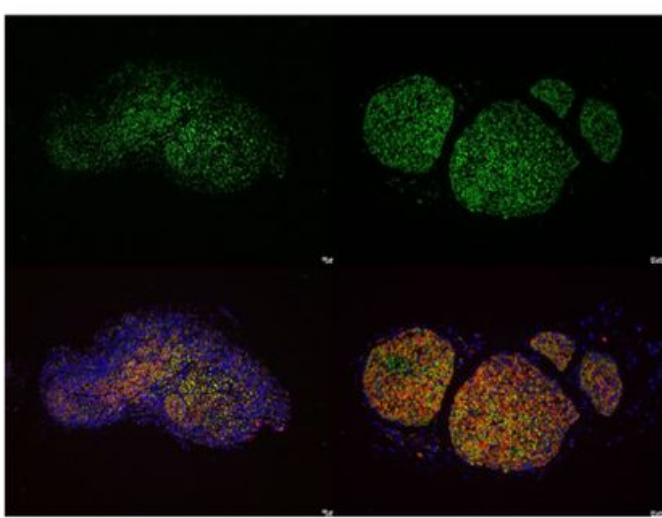

Reverse Orientation

Proximal

Center

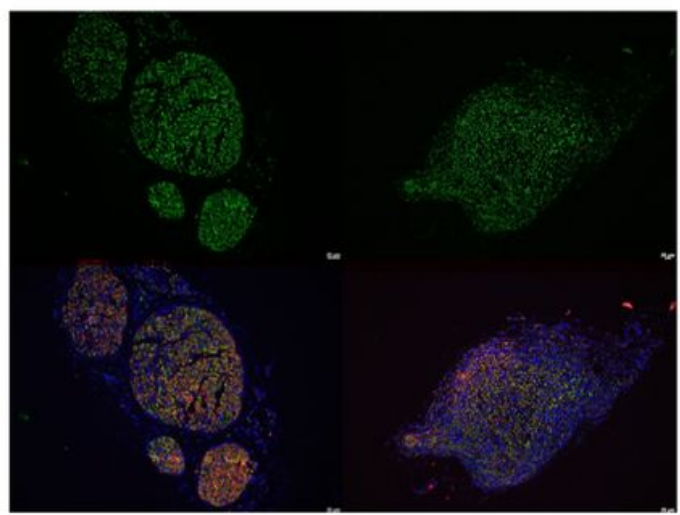

B
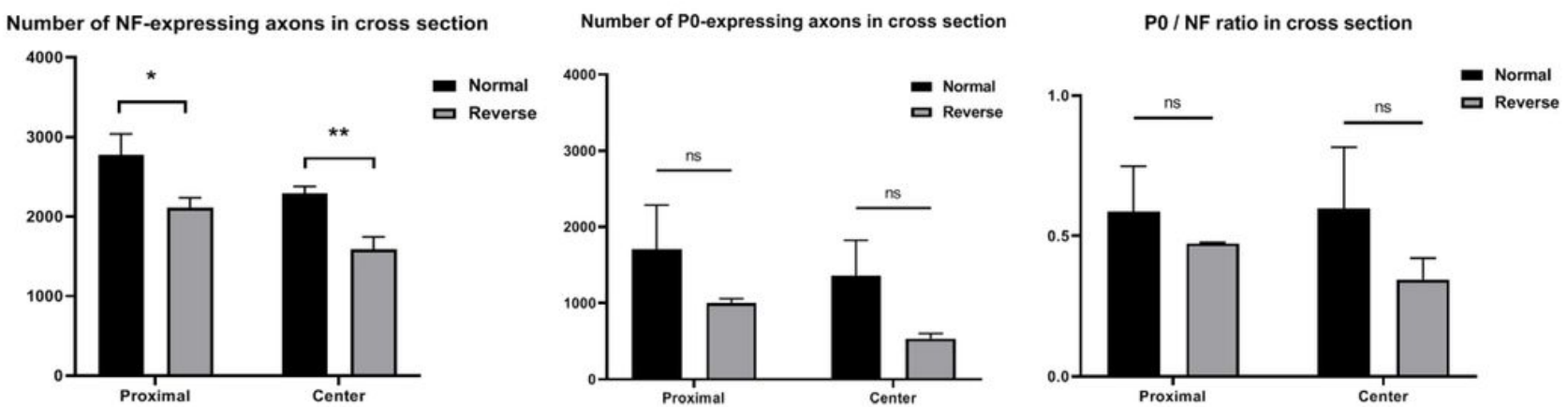

Figure 2

Representative cross-sectional images of the autograft and counts of NF- or P0-expressing axons in cross-sectional images. (A) Representative cross-sectional images of the nerve at the proximal and central autograft on immunofluorescence staining $(\times 200)$. Normally oriented autografting increased the total number of NF-expressing axons within the autograft. (B) The number of NF-expressing axons in normally oriented autografts was higher than that in reverse-oriented autografts in the proximal and central area. The number of P0-expressing axons in normally oriented autografts was higher than that in reverse-oriented autografts, and normally oriented autografts had a higher ratio of P0-/NF-expressing

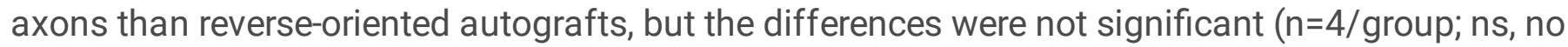
significance; ${ }^{*} \mathrm{P}<0.1$; $* * \mathrm{P}<0.05$; Green (Alexa Fluor 488 1:500, ab197485, Abcam), neurofilament (NF) heavy chain (1:500, ab4680, Abcam); Red (Alexa Fluor 594 1:500, ab150088, Abcam), myelin protein 0 (P0, 1:200, ab31851, Abcam); Blue (4',6-diamidino-2-phenylindole [DAPI] 1:1000, ab228549, Abcam); Normal, normally oriented autograft; Reverse, reverse-oriented autograft). 

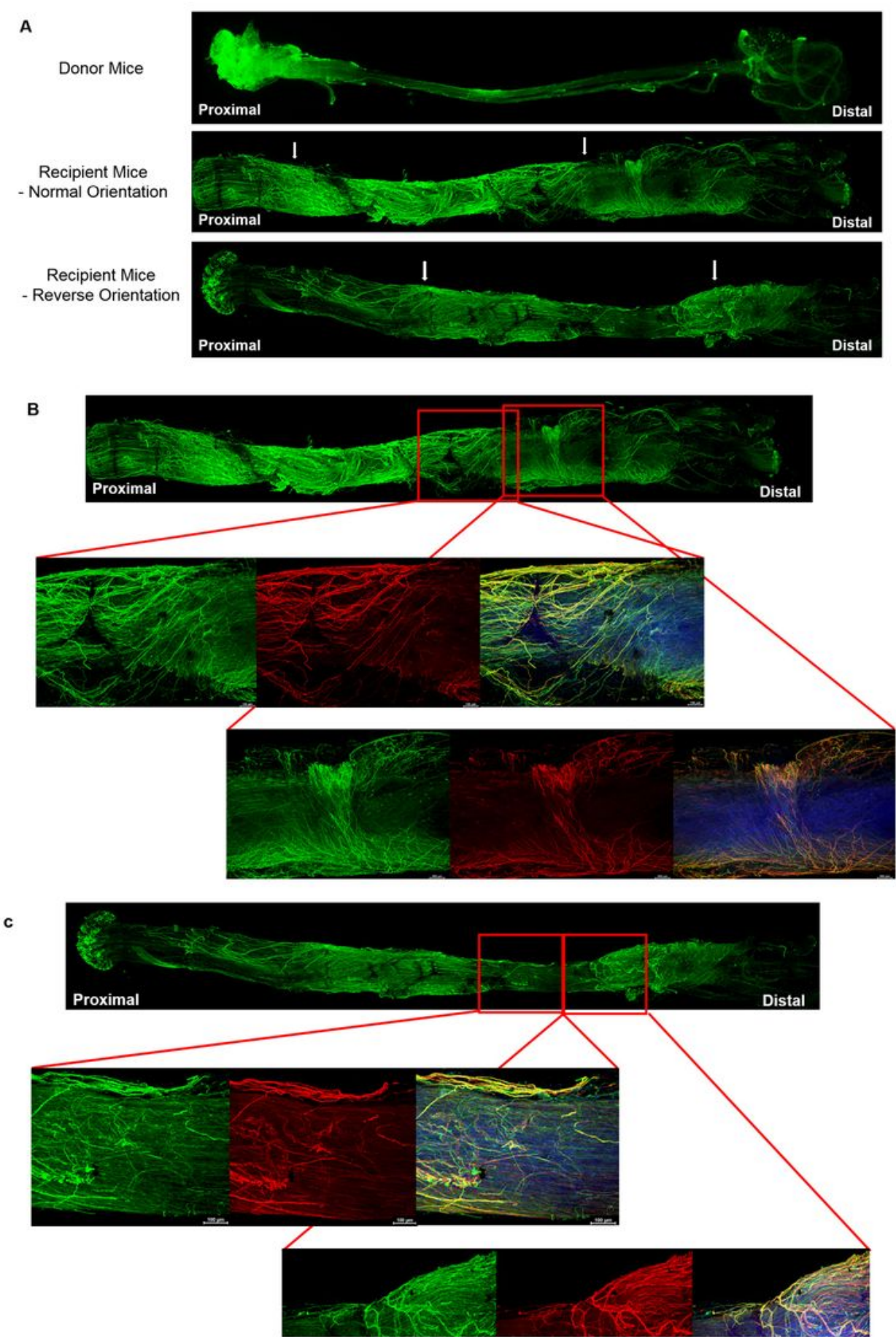

\section{Figure 3}

Representative immunofluorescence staining images of whole nerve, combining seven confocal microscopic images in donor and recipient mice. (A) Thin fibrous tissue containing some regenerating NF-expressing axons was observed in the empty space after the $10-\mathrm{mm}$ sciatic nerve was harvested in donor mice. The abundant NF-expressing axons regrew crossing the junctional area between the stumps and autograft in mice treated with normally and reverse-oriented autografts. (B) The distal junctional area 
between the autograft and distal stump of recipient nerve showed that numerous NF- or P0-expressing axons regrew multidirectionally and circumferentially in mice treated with normally oriented autografts. Blue-colored DAPI-positive cells were abundant inside the distal stump. (C) The distal junctional area showed that numerous NF- or P0-expressing axons regrew relatively longitudinally, crossing the junctional area toward the distal stump in mice treated with reverse-oriented autografts ( $n=5 / g r o u p$; white arrow, the junction between the stump and autograft; Green (Alexa Fluor 488 1:500, ab197485, Abcam), neurofilament (NF) heavy chain (1:500, ab4680, Abcam); Red (Alexa Fluor 594 1:500, ab150088, Abcam), myelin protein 0 (P0, 1:200, ab31851, Abcam); Blue (4',6-diamidino-2-phenylindole [DAPI] 1:1000, ab228549, Abcam)).

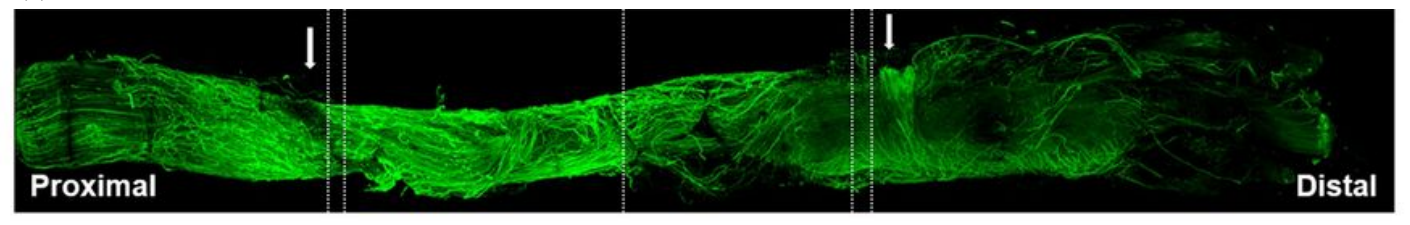

A. Number of NF-expressing axons in graft

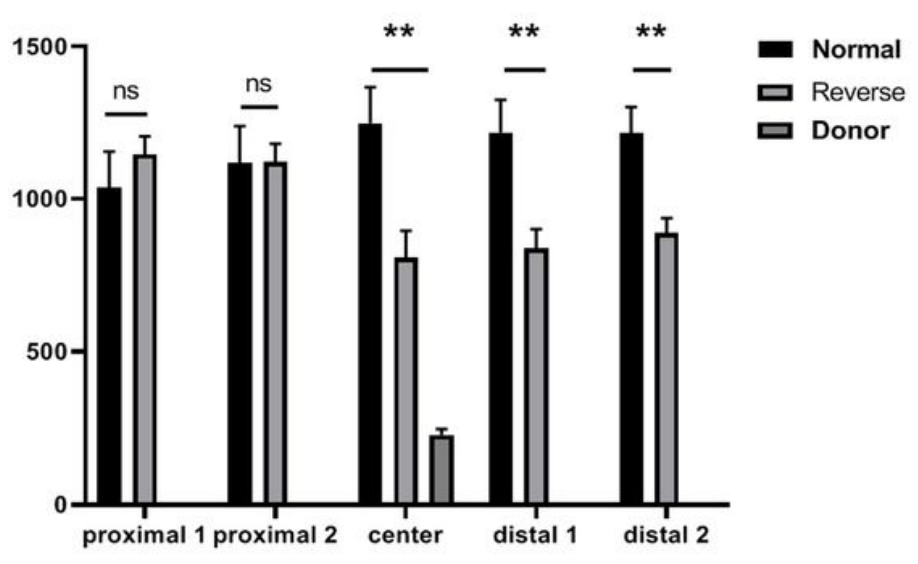

B. Mean signal of $\mathrm{P} 0$-expressing axons in whole mouting nerve

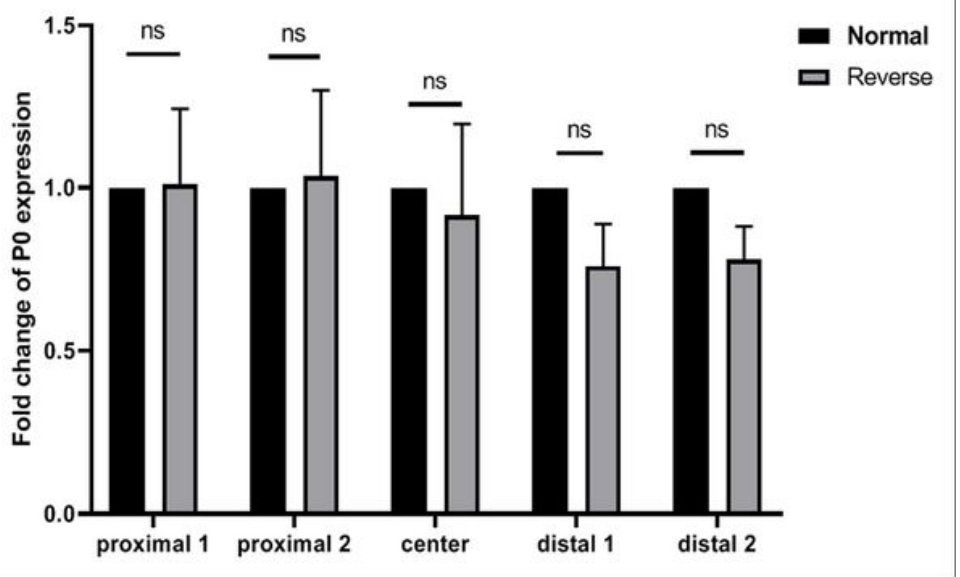

Figure 4

Counts of NF-expressing axons and mean fluorescence intensity $(\mathrm{FI})$ of $\mathrm{P} 0$-expressing axons on five lines -two proximal, one central, and two distal areas of the autograft. (A) Mice treated with normally oriented autograft had higher number of NF expressing axons inside and in the distal junction of autograft than those with the reverse-oriented autograft. (B) The mean FI of P0-expressing axons in the distal junction of autograft was higher in normally oriented autografts than in reverse-oriented autografts, but these differences were not significant ( $n=5$ /group; $n$, no significance; $N F$, neurofilament heavy chain;** $P<0.05$ ). 
A.
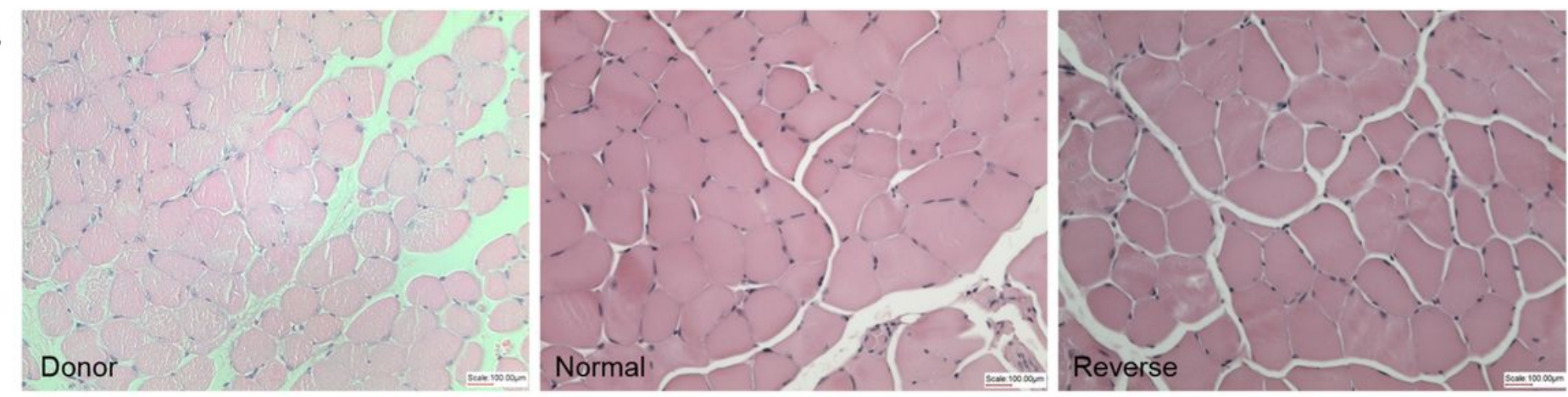

B. TA muscle weights ratio

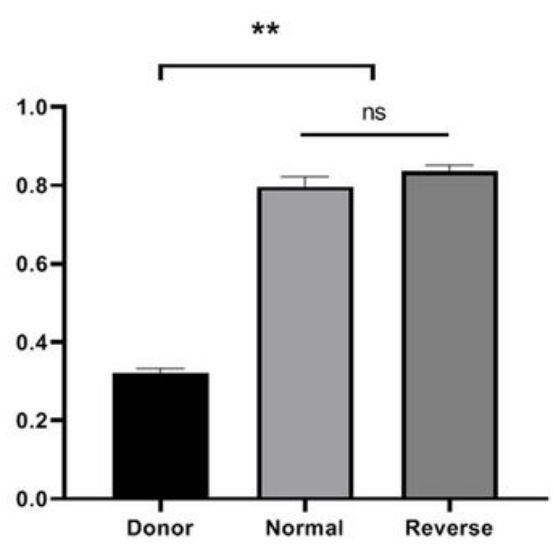

C. MFD of TA muslce $(\mu \mathrm{m})$

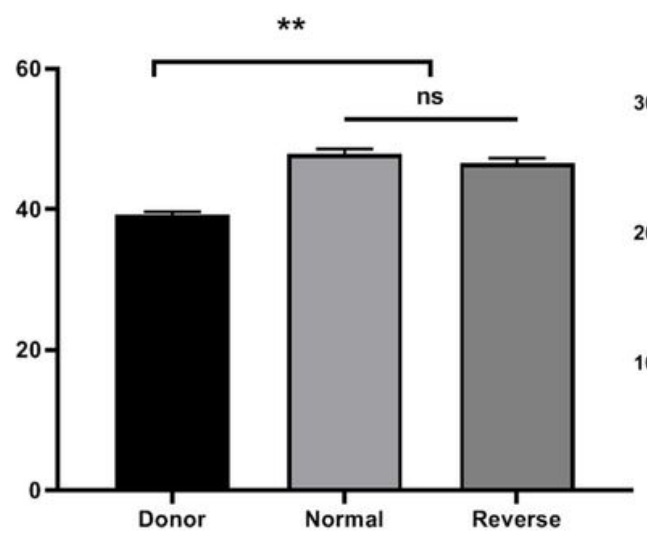

D. CSA of TA mucle $\left(\mu \mathrm{m}^{2}\right)$

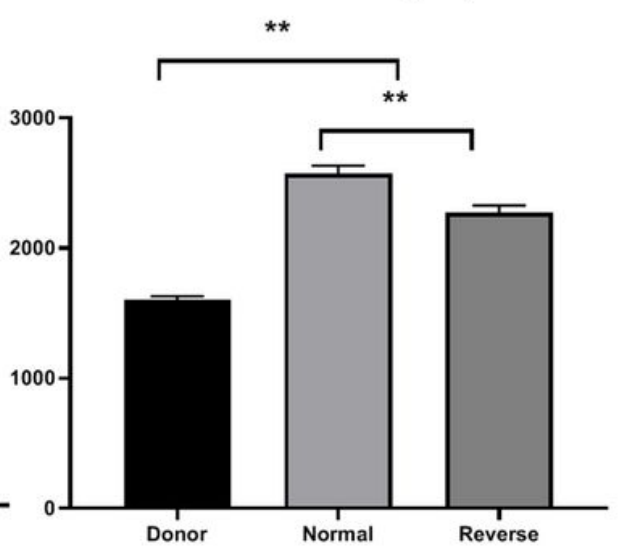

Figure 5

Quantitative analysis of denervation-induced atrophy of the TA muscle. (A) Representative H\&E images of transverse sections of the tibialis anterior $(T A)$ muscle $(\times 200)$. The TA muscle of donor mice showed decreased myofiber size and increased interfiber spacing. However, interpositional nerve grafting with normal or reverse orientation prevented these phenomena. (B, C, D) The wet weight ratio, minimum Feret's diameter (MFD), and cross-sectional area (CSA) of TA myofibers in mice treated with interpositional nerve grafting with normal or reverse orientation were higher than those in donor mice. $(B, C)$ The wet weight ratio and the MFD of myofibers was not significantly different between normally and reverse-oriented autografts. (D) The CSA of myofibers in mice treated with normally oriented autograft was larger than that in mice treated with reverse-oriented autograft ( $n=4$ /group; ns, no significance; ${ }^{\star \star} P<0.05$; Normal, normally oriented autograft; Reverse, reverse-oriented autograft) 
Donor Mice
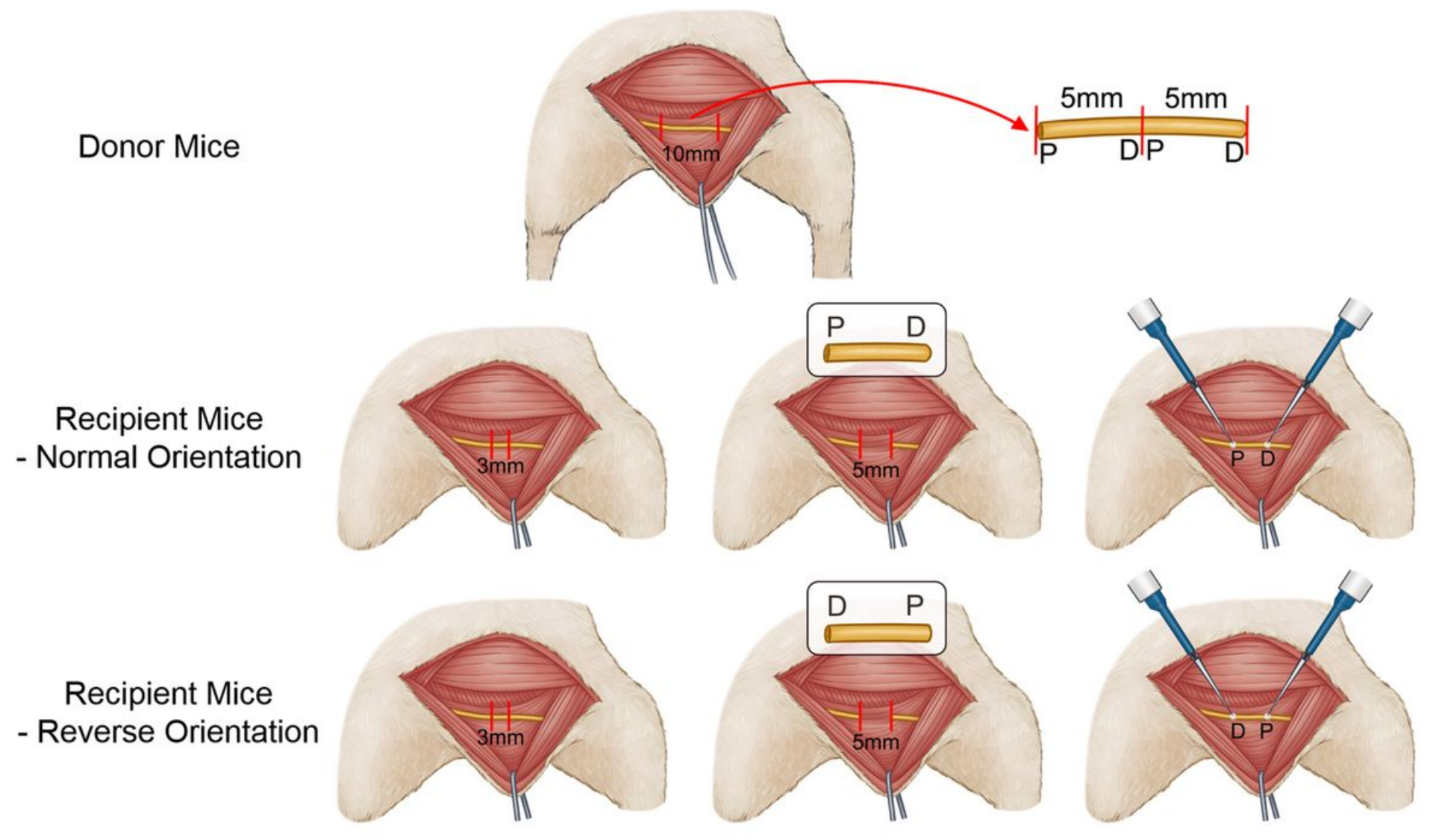

\section{Figure 6}

Schematic drawings explaining experimental design and surgical protocols. In donor mice, a 10-mm sciatic nerve was harvested from proximal (after passing through the sciatic foramen) to distal (before the trifurcation branching). In recipient mice, a 3-mm sciatic nerve was dissected for creating an irreparable nerve gap that retracts owing to elastic recoil, yielding a larger defect. Half the of autograft (5 $\mathrm{mm}$ ) was placed in the nerve gap of recipient mice, either in normal or reversed proximodistal orientation, in good alignment and coapted with fibrin sealant at both coaptation sites under an operating microscope (P: Proximal, D: Distal). 